\title{
Supernumerario invertido en paladar de un infante: reporte de un caso clínico
}

\section{Supernumerary inversion in the palate of an infant: report of a clinical case}

\author{
Wendolin Trejo-García', Martha Mendoza-Rodríguez ${ }^{1}$, Carlo Eduardo Medina-Solís ${ }^{1,2}$, \\ Miriam Alejandra Veras-Hernández', Salvador Eduardo Lucas-Rincón², Juan Fernando \\ Casanova-Rosado ${ }^{3}$
}

\section{RESUMEN}

Introducción: La hiperdoncia, es una anomalía de número en la que se forman dientes o estructuras parecidas a dientes en exceso con respecto a la cantidad normal, teniendo como resultado órganos dentales llamados supernumerarios. Presentación del caso: Paciente masculino de 7 años 11 meses de edad con dentición mixta y retraso en la erupción de los incisivos centrales y laterales superiores. En la radiografía panorámica se observa una zona radiopaca entre el incisivo central superior permanente (OD 21) y el incisivo lateral superior permanente (OD 22) diagnosticando un diente supernumerario. Con ayuda de una radiografía oclusal y una periapical de la zona se valoró y se determinó su extracción bajo anestesia local. Se realizó la cirugía sin complicaciones y se derivó al paciente a la clínica de ortodoncia. Conclusiones: En el presente reporte se pudo observar que llevando un buen control del caso y con la ayuda de estudios radiográficos se puede lograr un avance significativo sin dañar la integridad bucal del paciente en un futuro. Significancia clínica: El diagnóstico precoz puede mejorar con ayuda de los diversos tipos de radiografías disponibles en odontología, así se evitan complicaciones al paciente infantil.

Palabras Clave: Hiperdoncia, supernumerario, erupción invertida.

\begin{abstract}
Introduction: Hyperdontia is a number anomaly in which teeth or structures similar to teeth are formed in excess of the normal amount, resulting in supernumerary dental organs. Case presentation: a male patient 7 years 11 months of age with mixed dentition and delayed eruption of the central and upper lateral incisors presented for evaluation. The panoramic radiograph showed a radiopaque area between the permanent upper central incisor (OD 21) and the permanent upper lateral incisor (OD 22). A supernumerary tooth was diagnosed. With the help of an occlusal and a periapical radiograph of the area, the tooth was evaluated and an extraction under local anesthesia was planned. The surgery was performed without complications and the patient was referred to the orthodontic clinic. Conclusions: In the present report, we note that time intervention and with the help of radiographic studies, a significant result can be achieved without damaging the patient's future oral integrity. Early diagnosis can be improved with the help of the various types of radiographs available in dentistry, thus avoiding complications in infants.
\end{abstract}

Keywords: Hyperdontia, supernumerary, inverted eruption.

\footnotetext{
${ }^{1}$ Universidad Autónoma del Estado de Hidalgo, Instituto de Ciencias de la Salud, Área Académica de Odontología, . Pachuca, México.

${ }^{2}$ Universidad Autónoma del Estado de México, Facultad de Odontología, Centro de Investigación y Estudios Avanzados en Odontología “Dr. Keisaburo Miyata”. Toluca, México.

${ }^{3}$ Universidad Autónoma de Campeche, Facultad de Odontología. Campeche, México.

Correspondencia: Carlo Eduardo Medina Solís Correo: cemedinas@yahoo.com

Conflicto de interés: Los autores declaran no tener conflicto de intereses.

Recibido: 5/12/2017 Aceptado: 27/07/2018

DOI: https://doi.org/10.31698/ped.45032018008
} 


\section{INTRODUCCIÓN}

La odontogénesis, se refiere a la serie de procesos fisiológicos de crecimiento, estadios de formación y cambios morfológicos que se realizan para la formación de los dientes. En éste se distinguen dos fases: la morfogénesis o morfodiferenciación y la histogénesis o citodiferenciación) $)^{(1,2)}$. Durante estos procesos, pueden observarse diversas anomalías de forma, tamaño, número y estructura que deben ser bien conocidos por el pediatra y el odontopediatra. La hiperdoncia, es una anomalía de número en la que se forman estructuras dentales en exceso, teniendo como resultado órganos dentales llamados supernumerarios ${ }^{(1,3)}$. Los dientes supernumerarios pueden estar presentes en cualquier área de las arcadas de ambas denticiones, pero son 5 veces menos frecuentes en la dentición primaria. Tienen una prevalencia que oscila entre el 0.1 y el $3.6 \%$ en la dentición permanente, y entre 0.3 y 0.8 en la dentición primaria, observándose variaciones de acuerdo a la raza. Los hombres se ven afectados aproximadamente el doble de frecuencia que las mujeres $^{(3,4)}$. En México, se ha observado que la prevalencia de dientes supernumerarios es de $3.2 \%{ }^{(5)}$.

Los dientes supernumerarios son idiopáticos, se los puede asociar con varios síndromes y observarse en población no-sindrómica. Dado que la causa de los dientes supernumerarios muestra una heterogeneidad considerable, es menos probable que ciertos hallazgos sean coincidentes, como cinco o más dientes supernumerarios en un solo paciente o lugares fuera del premaxilar ${ }^{(6)}$. En general, la etiología es poco conocida. La teoría filogenética, la hiperactividad de la lámina dental, la dicotomía de la lámina dental y la influencia de los factores ambientales se informan como posibles mecanismos de formación de supernumerarios. La predisposición genética y hereditaria, pueden jugar también un papel importante ${ }^{(7)}$.

Los supernumerarios pueden ser clasificados de acuerdo a su tipo y forma. Las variaciones de tipo incluyen mesiodens, parapremolares, paramolares, $\mathrm{y}$ distomolares. La forma de supernumerarios varía desde un odontoma simple, a través de un diente cónico o tuberculado hasta un diente suplementario que se parece mucho a un diente normal ${ }^{(5)}$. Además, el número y el sitio de los supernumerarios puede variar considerablemente, pueden ocurrir solo o múltiple, unilateral o bilateralmente, y en uno o ambos maxilares. Los casos que involucran uno o dos supernumerarios involucran con mayor frecuencia el maxilar anterior, seguido de la región premolar mandibular ${ }^{(3-5)}$. Los dientes supernumerarios pueden causar diferentes trastornos locales relacionados con la oclusión, como prolongada retención de dientes primarios, erupciones ectópicas, desplazamientos dentales, apiñamiento, erupción retardada de los dientes permanentes, impactación dental, anomalías espaciales; resorción radicular de dientes adyacentes, además de patologías como quistes foliculares y otras alteraciones que requieren una intervención quirúrgica o ortodóncica ${ }^{(3-7)}$. El tratamiento depende del tipo y la posición del diente supernumerario y su efecto potencial sobre los dientes adyacentes. De igual forma, las modalidades de tratamiento pueden variar desde la detección temprana a la eliminación, lo que permite la resolución espontánea de la irregularidad oclusal a la corrección mediante un enfoque de equipo multidisciplinario. El tratamiento debe formar parte de un plan de tratamiento integral y no debe considerarse de forma aislada ${ }^{(3-5)}$.

\section{CASO CLÍNICO}

Paciente masculino de 7 años 11 meses de edad, acude a la clínica de odontopediatría de la Licenciatura de Cirujano Dentista de la Universidad Autónoma del Estado de Hidalgo (Pachuca, México). Se inició con la exploración intraoral, pudiendo observar la forma de los arcos que en superior es ovalada y en inferior cuadrada. El paciente presentaba dentición mixta, con caries en órganos dentales (OD) 64, 74, 84 y 85, así como restauraciones de coronas de acero-cromo en 54 y 55 . Se observó la ausencia de los incisivos centrales y laterales permanentes superiores. Como presentaba apiñamiento en el arco inferior, también se remitió a la clínica de ortodoncia. Se solicitó una radiografía panorámica para evaluar la correcta erupción de los dientes faltantes, así como también valorar a los demás órganos dentarios en su totalidad y corregir el apiñamiento. Como resultado de la radiografía se observó una zona radiopaca entre el incisivo central 
superior permanente (OD 21) y el incisivo lateral superior permanente (OD 22) diagnosticando un diente supernumerario. Se solicitó estudios más completos para determinar la localización del diente supernumerario, ya que su posición se observaba invertida desde un inicio. Con ayuda de una radiografía oclusal y una periapical de la zona se valoró y se determinó su extracción (Figura 1).

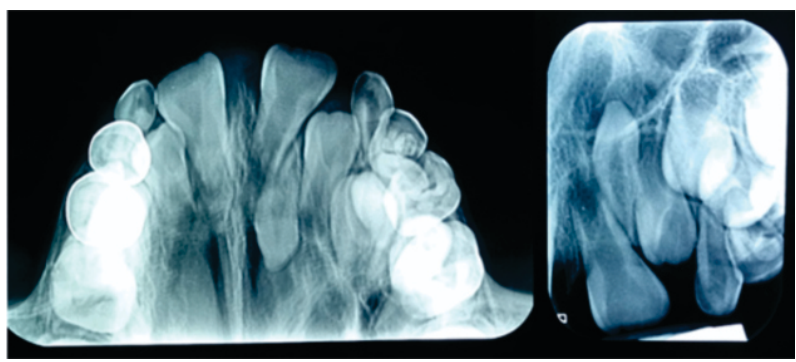

Figura 1. Radiografía oclusal y radiografía periapical. Fuente: Elaboración propia de los autores.

\section{Procedimiento quirúrgico}

Se llevó a cabo la cirugía comenzando con la asepsia y antisepsia de la zona operatoria, se procedió a infiltrar Lidocaína al 2\% con epinefrina 1:100,000 en los nervios alveolar posterior y palatina anterior, así como puntos locales. El abordaje fue realizado por palatino para liberar encía que cubría al diente supernumerario, se levantó el colgajo mucoperiostico y de ésta manera se obtuvo una visión del campo operatorio sobre el diente el cual estaba dentro del paladar, se realizó osteosección de la periferia del órgano dental, irrigando con suero fisiológico, y se aplicó la luxación con la ayuda de los elevadores, se hizo la exodoncia propiamente dicha y se limpió el lecho quirúrgico con abundante suero fisiológico, se revisó la hemostasia, se aplicaron 2 spongostand y se procedió a afrontar el tejido suturando la zona con Vycril 000 comenzando por todo el abordaje, es decir, entre las papilas dentarias, se colocaron 3 puntos, se dio farmacoterapia y se finalizó el tratamiento sin complicaciones. Se citó al paciente 8 días después de la cirugía para concluir con el retiro de puntos (Figura 2). La Figura 3 muestra el diente supernumerario extraído. La Figura 4 muestran que los incisivos centrales entraron en erupción hacia la cavidad bucal, y la radiografía periapical muestra un buen resultado.

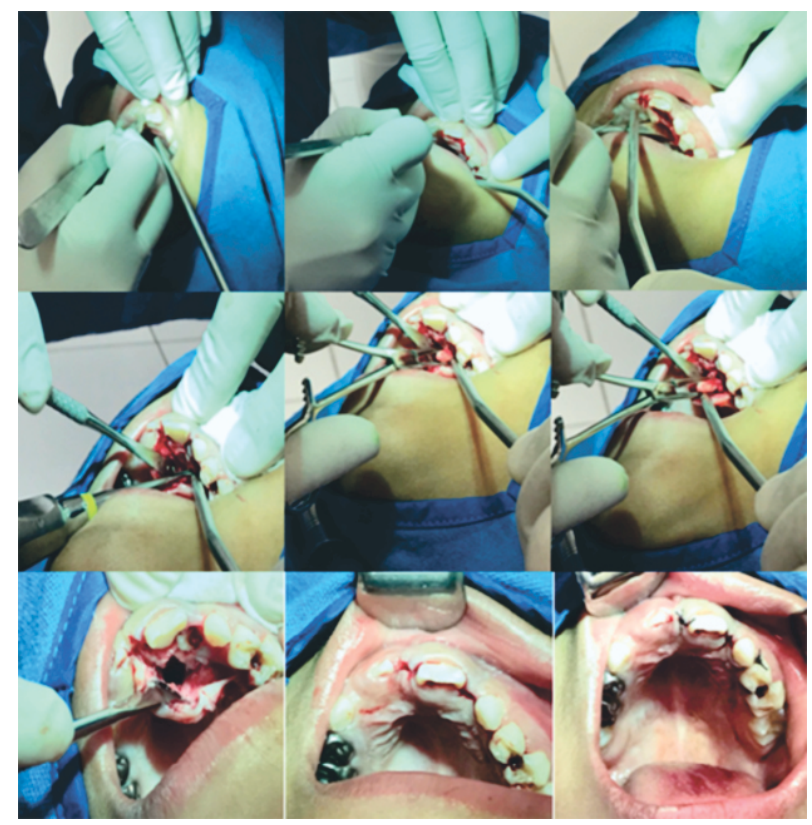

Figura 2. Procedimiento quirúrgico.

Fuente: Elaboración propia de los autores.

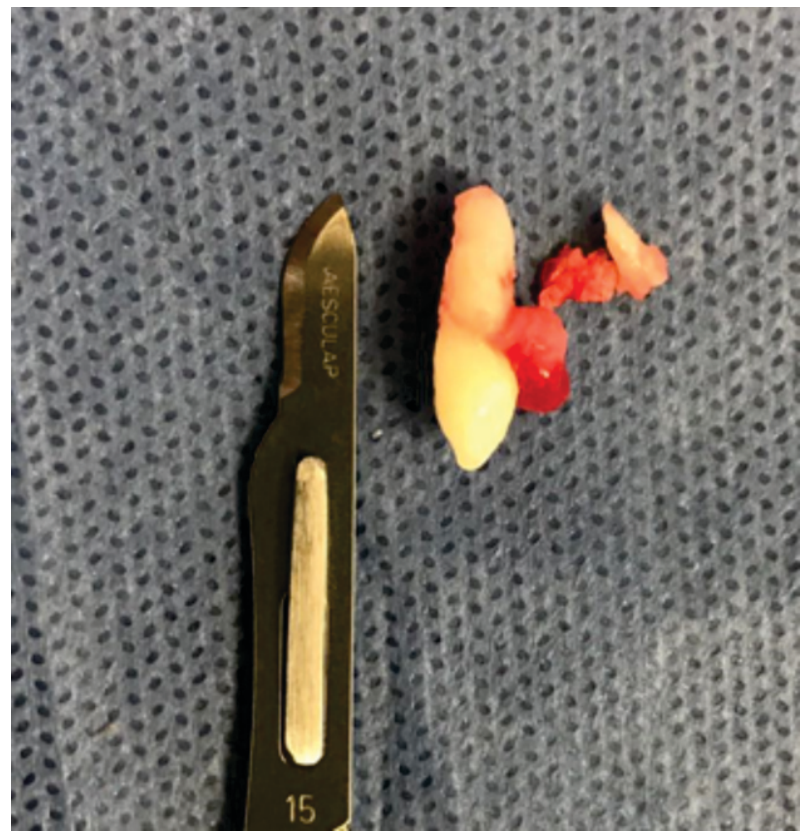

Figura 3. Diente supernumerario extraído.

Fuente: Elaboración propia de los autores. 


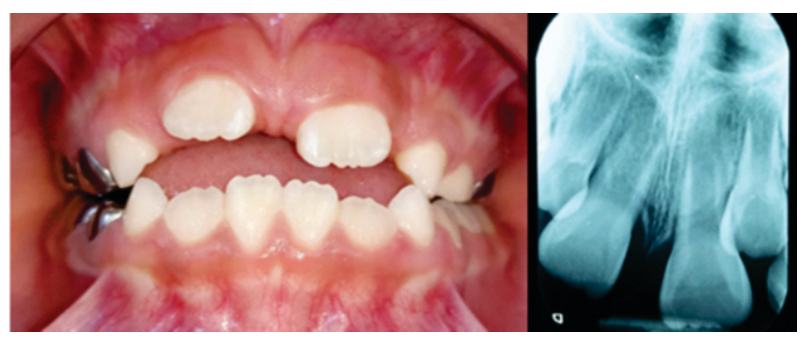

Figura 4. Fotografía final a las 5 semanas. Radiografía periapical después de 5 semanas de realizada la cirugía.

Fuente: Elaboración propia de los autores.

\section{Procedimiento ortodóntico}

En la clínica de ortodoncia se confeccionaron 2 placas tipo Hawley, superior e inferior, con tornillo de expansión bilateral, arco vestibular elaborado con alambre del calibre $0.32 \mathrm{~mm}$, así como ganchos de bola del calibre $0.28 \mathrm{~mm}$ colocados entre el segundo molar deciduo y el primero molar permanente y una base acrílica de color verde. Se le entregaron indicaciones del cuidado de sus aparatos ortopédicos y se le realizaron las activaciones pertinentes cada 8 días activando $1 / 4$ de vuelta a cada uno.

\section{DISCUSIÓN}

Cualquier retraso en la erupción, erupción asimétrica o rotación de un órgano dental debe alertarnos de la posible existencia de un diente supernumerario, tal y como ocurrió en el caso presentado, donde se observó una alteración en la secuencia de erupción. Por lo que sólo el diagnóstico precoz evitará estas complicaciones y nos permitirá realizar el tratamiento más adecuado, que en este caso fue la extracción del diente supernumerario, y de esta forma evitar daños futuros. En diversas situaciones se presentan tratamientos de acuerdo a la edad en la que aparezca el diente supernumerario, por lo cual existen dos momentos. La extracción temprana del supernumerario se realiza en cuanto se diagnostica, incluso antes de los 6 años. Se prefiere este momento para prevenir futuros problemas, como erupción ectópica o retraso en la erupción del incisivo permanente. Otros autores prefieren la exodoncia tardía del supernumerario. La extracción se realiza cuando se ha completado la formación radicular de los incisivos permanentes adyacentes. El mayor inconveniente es que cuanto más tarde se haga la extracción, el ápice del diente impactado estará más cerrado y es más probable necesitar un tratamiento quirúrgico y ortodóntico posterior ${ }^{(8)}$. El tratamiento combinado ortodóncico quirúrgico, tal como se muestra en el caso, se ha convertido en una de las mejores opciones. Esto soluciona los problemas periodontales y estéticos tras la impactación de un incisivo a consecuencia de un supernumerario en vez de reposicionamientos quirúrgicos del diente permanente.

Es importante que los cirujanos dentistas, odontopediatras, cirujanos bucales y maxilofaciales, médicos pediatras, e incluso los antropólogos, genetistas y otros profesionales de la salud conozcan la prevalencia de anomalías dentales en diferentes comunidades. Los auxiliares de diagnóstico, como las radiografías, deben ser explotados para ayudar en el diagnóstico clínico y prevenir complicaciones futuras. La radiología es un medio auxiliar fundamental en odontopediatría y cirugía bucal, que complementa el estudio clínico del paciente. Las radiografías confirman muchos de los datos observados en la exploración clínica y revelan otros nuevos de una forma rápida y efectiva. Los dientes supernumerarios no erupcionados pueden ser diagnosticados con una radiografía panorámica, y mejorar la localización con ayuda de las radiografías periapical y oclusal. En la mayoría de los casos, los dientes supernumerarios están impactados y sólo pueden ser visibles radiográficamente ${ }^{(9)}$.

\section{CONCLUSIONES}

Se pudo observar que con la ayuda de estudios radiográficos se puede lograr un avance significativo sin dañar la integridad bucal del paciente. Para la correcta erupción de los dientes permanentes el tratamiento ortodóntico es importante, ya que podremos devolver la función y estética al paciente. 


\section{REFERENCIAS BIBLIOGRÁFICAS}

1. Vastardis $\mathrm{H}$. The genetics of human tooth agenesis: new discoveries for understanding dental anomalies. Am J Orthod Dentofacial Orthop. 2000;117:650-6.

2. Lucas-Rincón SE, Medina-Solís CE, Pontigo-Loyola AP, Robles-Bermeo ML, Lara-Carrillo E, Veras-Hernández MA et al. Natal and neonatal teeth: a review of the literature. Pediatr (Asunción). 2017;44:62-70.

3. Omami M, Chokri A, Hentati H, Selmi J. Cone-beam computed tomography exploration and surgical management of palatal, inverted, and impacted mesiodens. Contemp Clin Dent. 2015;6(Suppl 1):S289-93.

4. Burhan AS, Nawaya FR, Arabi Katbi ME, Al-Jawabra AS. Prevalence of supernumerary teeth in a nonsyndromic Syrian sample. J Egypt Public Health Assoc. 2015;90(4):146-9.

5. Salcido-García JF, Ledesma-Montes C, HernándezFlores F, Pérez D, Garcés-Ortíz M. Frecuencia de dientes supernumerarios en una población Mexicana. Med Oral Patol Oral Cir Bucal. 2004;9:403-9.

6. Lubinsky M, Kantaputra PN. Syndromes with supernumerary teeth. Am J Med Genet A. 2016;170(10):2611-6.

7. McBeain M, Miloro M. Characteristics of Supernumerary Teeth in Nonsyndromic Population in an Urban Dental School Setting. J Oral Maxillofac Surg. 2018;76(5):933-938.

8. Fernández-Miñano E, Hernández-Fernández A, LucasPenalva P, Ortiz-Ruiz AJ. Manejo ortodoncicoquirúrgico de un incisivo central superior impactado debido a un mesiodens Odontol Pediátr (Madrid). 2015;23(3):210-218.

9. Ephraim R, Dilna NC, Sreedevi S, Shubha M. A labially positioned mesiodens and its repositioning as a missing central incisor. J Int Oral Health. 2014;6(5):114-117. 